\title{
Luminescence spectroscopy and imaging: analytical advances and perspectives in the Earth sciences and related disciplines
}

\author{
Lutz Nasdala • Jens Götze • John M. Hanchar
}

Received: 15 April 2013 / Accepted: 22 April 2013 / Published online: 8 May 2013

(C) Springer-Verlag Wien 2013

This special issue is devoted to review articles on various themes and topics related to the application of luminescence spectroscopy and luminescence-based imaging in the Earth sciences and related disciplines. It was compiled on the occasion of the CORALS-2013, the third Conference on Raman and Luminescence Spectroscopy in the Earth sciences (July 3-6, 2013, Vienna, Austria).

In contrast to the two previous CORALS conferences, held in Mainz, Germany (2009), and Madrid, Spain (2011), the CORALS-2013 was not focused preferentially on research in Lunar and planetary sciences, but it was open explicitly to applications of Raman and luminescence spectroscopy in all fields of the Earth sciences and related disciplines. In view of its broad profile as regards scientific content, the conference therefore seemed to be an excellent occasion to summarise the current state of the research. This, however, has already been done just recently for the Raman technique: A wide range of applications of Raman spectroscopic analyses has been presented comprehensively in volume 12 of the EMU Notes in Mineralogy (J. Dubessy, M.-C. Caumon, F. Rull, Eds.:

\section{Editorial handling: R. Abart}

L. Nasdala $(\bowtie)$

Institut für Mineralogie und Kristallographie,

Universität Wien, Althanstr. 14,

1090 Wien, Austria

e-mail: lutz.nasdala@univie.ac.at

\section{J. Götze}

Institut für Mineralogie, TU Bergakademie Freiberg,

Brennhausgasse 14,

09596 Freiberg/Sa., Germany

e-mail: goetze@mineral.tu-freiberg.de

J. M. Hanchar

Department of Earth Sciences,

Memorial University of Newfoundland,

Alexander Murray Building, 300 Prince Philip Drive,

St. John's, NL A1B 3X5, Canada

e-mail: jhanchar@mun.ca
Applications of Raman spectroscopy to Earth sciences and cultural heritage. European Mineralogical Union, 2012), and the use of Raman-based images in the Earth sciences has been summarised in an extended review chapter contained in volume 168 of Springer Series in Optical Sciences (A. Zoubir, Ed.: Raman imaging - Techniques and applications. Springer, Berlin Heidelberg, 2012). This special issue therefore focuses on luminescence techniques only.

Other than most special issues related to conferences, this present issue dos not consist of a selection of research contributions that were presented during the conference. Rather, a number of review articles were invited well in advance, with the aim to compile a summary of the current status of luminescence seen from the Earth-sciences perspective, by the time the conference starts. Not all of our invitations were accepted, and not all of the submissions received could be published. For these reasons, several important fields (such as ion-beam luminescence, dosimetry based on afterglow-type luminescence, or the use of internal textures of minerals observed in luminescence images to assist isotope geochronology techniques) are not covered here; the summary hence must remain incomplete. We are nevertheless convinced that the eight review papers in this volume present a well-balanced selection of articles documenting the broad range of recent research activities and advances. To underline and illustrate the versatility of luminescence techniques in studying geological samples and synthetic minerals, we present a number of spectra and images in Fig. 1. We feel, however, obliged to add that at present, applications of luminescence spectroscopy and imaging applied in the Earth sciences are way more versatile than it can be visualised in a single paper or even a single figure. This assessment is supported by the range of topics presented in the present issue.

Barbin contributed a summary of the application of the cathodoluminescence (CL) technique to the study of biogenic materials. As an example, the CL emission of trace-Mn in carbonate minerals is discussed, whose incorporation mechanism depends on the primary growth 

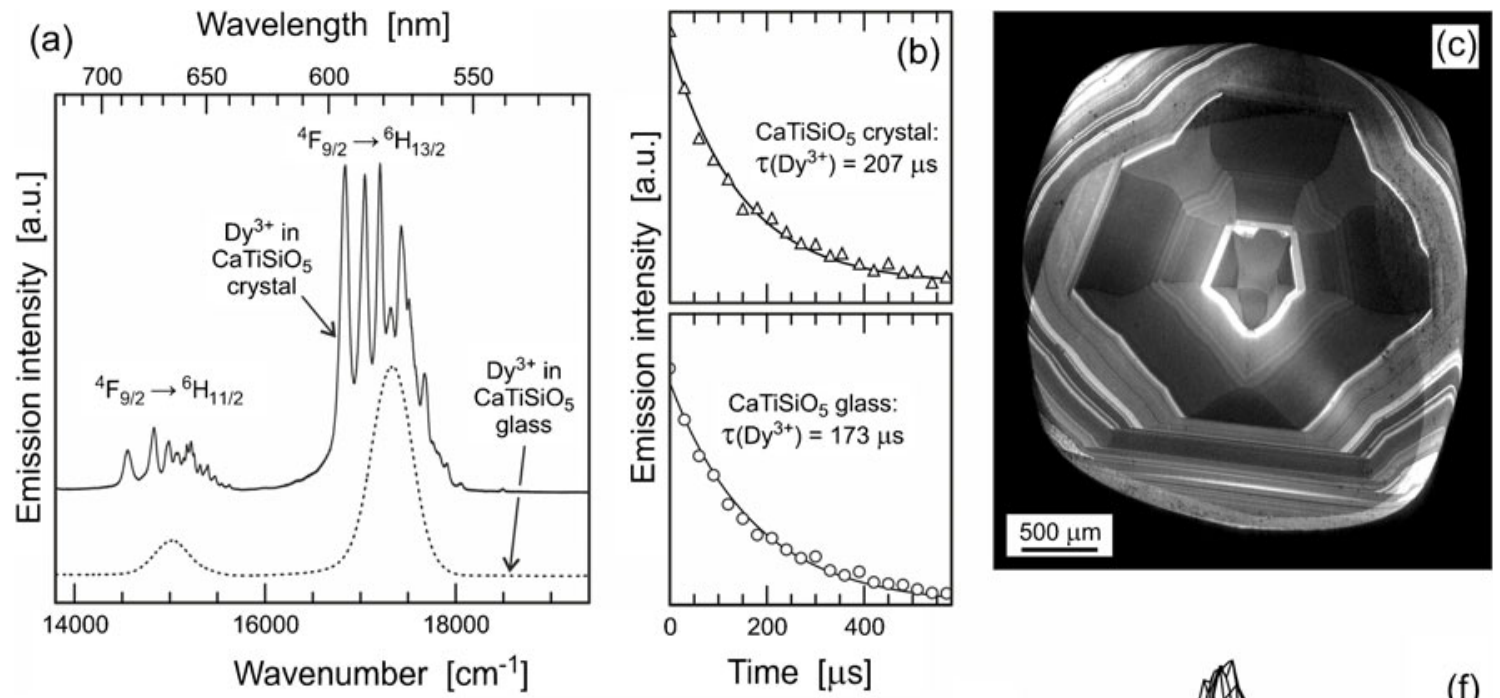

(d)
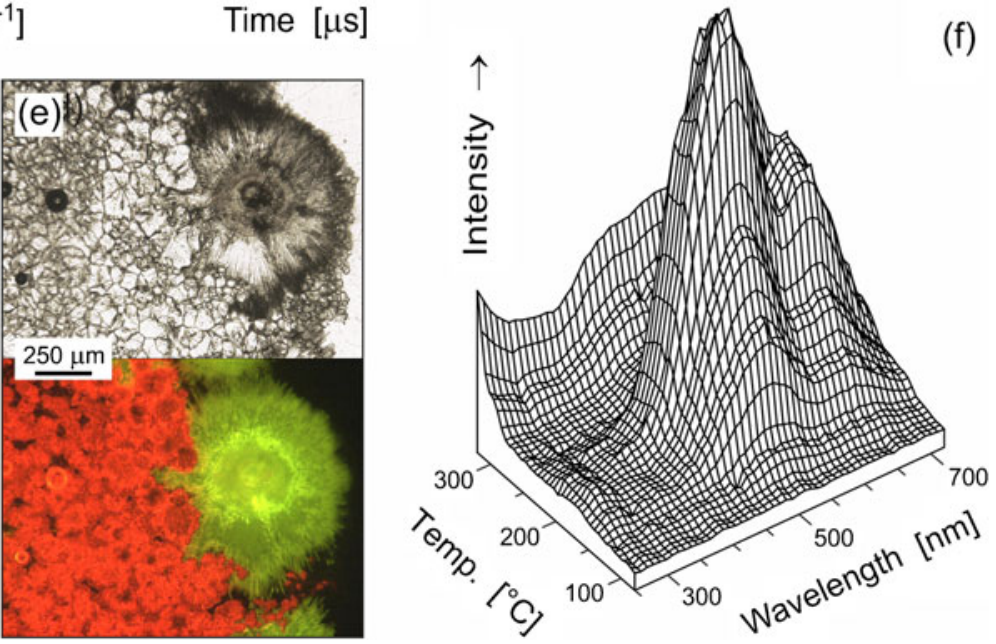

Fig. 1 Examples for the versatile application of luminescence spectra and images. a Steady-state PL spectra of synthetic Dy-doped titanite crystal and glass, visualising the splitting of main electronic transitions due to crystal-field effects. b Decay curves derived from time-resolved $\mathrm{PL}$, visualising different lifetimes of the excited stage of $\mathrm{Dy}^{3+}$. Data courtesy G. Panczer. c SEM-CL image of a mixed-habit diamond from Wellington, SE Australia, revealing growth and sector zoning. Image

conditions and the history of the host material. The Mnactivated luminescence may therefore be used, for instance, for a "provenance assignment" of shells (i.e. characterisation of their growth milieu) or the characterisation of archaeological fireplaces. Gaft and Panczer demonstrate the advantages of time-resolved over conventional steady-state luminescence in identifying defect centres present in minerals, and they summarise the new findings made during the last ten years. In addition they present a relatively new technique, namely plasma-induced luminescence, and discuss its possible uses and applications. The paper by Götze et al. is virtually an atlas of internal textures of rock-forming and accessory minerals, which are revealed most sensitively by the optical-microscope cathodoluminescence (OM-CL). This technique is most useful in unravelling growth, alteration, and other internal patterns that prove useful in interpreting growth courtesy D. Howell. d,e CL spectra and OM-CL images of a synthetic intergrowth of Mn-bearing carbonates. The different CL colours of aragonite (green) and calcite (orange-red) are explained by the shift of the main $\mathrm{Mn}^{2+}$ emission band. Courtesy C. Lenz. f Three-dimensional TL spectrum of quartz from a Quaternary sand sample, consisting of a grid of emission spectra (right abscissa) and glow curves (left abscissa). Data courtesy M. Krbetschek (deceased)

conditions and the post-growth history. Götze et al. verify with a wealth of impressive examples that the combined application of CL imaging and spectroscopy provides a much better understanding of the real structure of minerals, including their structural state and the incorporation of non-formula elements. Hainschwang et al. demonstrate an excellent combination of well-established spectroscopy research and commercial application in the field of gem testing. After a summarising overview of photoluminescence (PL) spectroscopy and imaging of coloured gems (also covering pearls and other biogenic materials), these authors show for the example of natural Type Ib diamond that, and how, luminescence spectroscopy is used to distinguish natural from synthetic specimens and to unravel colour enhancement by high pressure - high temperature treatment. The article by Lenz et al. focuses on effects of the crystal field on emissions of rare- 
earth elements (REEs). These authors demonstrate clearly that luminescence emissions (including CL emissions whose excitation is not polarised) show strong orientation effects. For the example of a radiative $\mathrm{Nd}$ transition, Lenz et al. show that REE-related emissions allow one to identify the host mineral and characterise its real structure. They finally conclude that REE-related emissions may serve as valuable "structural probes". MacRae et al. discuss new analytical opportunities arising from the application of hyperspectral CL in the microanalysis of minerals. Short acquisition times result in minimised electron-beam damage, compared to conventional imaging techniques. Mathematical data analysis nevertheless makes it possible to resolve minute compositional and growth textures, for instance those caused within single grains of quartz by external alpha irradiation and heterogeneous incorporation of trace-Ti, respectively. The article of Nasdala et al. summarises changes of the luminescence emission upon accumulation of irradiation damage. After an introductory summary of how corpuscular irradiation results in the accumulation of defects in minerals, the two apparently contrary effects of structural damage on the emission behaviour are discussed. While low levels of damage are occasionally associated with the generation of new emission centres, elevated levels of damage typically result in dramatic depletion of the luminescence intensity. Both effects were observed in a PL study of ionirradiated monazite, and it turned out that widths of certain $\mathrm{Nd}$ emission bands reflect most sensitively the degree of damage. Lastly, the paper by Stevens-Kalceff describes the use of $\mathrm{CL}$ techniques in studying silica polymorphs. In a study focussing on fused quartz and silica glasses, it is demonstrated that $\mathrm{CL}$ enables one to identify certain types of defects (such as oxygen vacancies, non-bridging oxygen defects, self-trapped excitons, or Al-related defects), and to conclude about the polymorph present, because spectral peculiarities of individual bands differ among polymorphs. In addition, effects of electron-beam damage are discussed in detail.

This special issue on luminescence could not have been accomplished without the constructive input of competent experts. We kindly thank the following people, who spent a lot of time and effort on reviewing contributions: Sanda Botis, Paul R. Edwards, Adrian Finch, Hans-Joachim Fitting, Javier Garcia-Guinea, Jaromir Leichmann, Roger Mason, Hanumanthappa Nagabhushana, Daniel Ohnenstetter, Karl Ramseyer, Dirk K. Richter, Andy H. Shen, Christopher L. Smith, Marion Stevens-Kalceff, Alexander M. Zaitsev, and Maria V. Zamoryanskaya. We also thank Rainer Abart and Anton Beran for their editorial help. 